\title{
Confined quantum Zeno dynamics of a watched atomic arrow
}

\author{
Adrien Signoles ${ }^{\dagger}$, Adrien Facon ${ }^{\dagger}$, Dorian Grosso, Igor Dotsenko, Serge Haroche ${ }^{\star}$, \\ Jean-Michel Raimond, Michel Brune and Sébastien Gleyzes
}

In a quantum world, a watched arrow never moves. This is the quantum Zeno effect'. Repeatedly asking a quantum system 'are you still in your initial state?' blocks its coherent evolution through measurement back-action. Quantum Zeno dynamics (QZD; refs 2,3) gives more freedom to the system. Instead of pinning it to a single state, it sets a border in its evolution space. Repeatedly asking the system 'are you beyond the border?' makes this limit impenetrable. As the border can be designed by choosing the measured observable, QZD allows one to dynamically tailor the system's Hilbert space. Recent proposals, particularly in the cavity quantum electrodynamics context $^{4,5}$, highlight the interest of QZD for quantum state engineering tasks ${ }^{6-11}$, which are the key to quantum-enabled technologies and quantum information processing. We report the observation of QZD in the 51-dimensional Hilbert space of a large angular momentum $J=25$. Continuous selective interrogation limits the evolution of this angular momentum to an adjustable multi-dimensional subspace. This confined dynamics leads to the production of non-classical 'Schrödinger cat' states $^{12,13}$, quantum superpositions of angular momenta pointing in different directions. These states are promising for sensitive metrology of electric and magnetic fields. This QZD approach could also be generalized to cavity and circuit quantum electrodynamics experiments ${ }^{4,5,13}$ by replacing the angular momentum with a photonic harmonic oscillator.

Quantum Zeno dynamics modifies the classical motion of a system by introducing a impenetrable barrier in the Hilbert space $^{4-11}$. This barrier can be equivalently induced by repeating a projective quantum measurement, performing a selective pulsed unitary acting on the states at the border ('Bang Bang' control) or even by applying a strong continuous coupling to these states ${ }^{3}$, as verified in a recent experiment ${ }^{14}$. In that experiment, however, the evolution of the system was restricted to a two-dimensional subspace. The dynamics was simply that of a spin $1 / 2$, and did not exhibit the most striking features of QZD (ref. 4).

In this Letter we implement QZD in a large atomic angular momentum $J=25$ ('spin' or top), represented as an arrow pointing on a generalized Bloch sphere. In the 51-dimensional Hilbert space, we isolate tailorable multi-dimensional manifolds. We show how QZD induces a very non-classical dynamics inside the Zeno subspace, leading to the generation of Schrödinger cat spin states $^{12}$, in which the arrow points at the same time in two different directions. As spin-squeezed states ${ }^{15}$, which are the focus of intense attention, these cat states lead to quantum-enabled metrological applications ${ }^{13}$.

The angular momentum projection on the polar axis of the generalized Bloch sphere is quantized, taking the values $J-k$, with $k=0 \ldots 2 J$ (the corresponding eigenstates being $|J, J-k\rangle$ ). The dynamical evolution from the initial state $|J, J\rangle$ (north pole of the Bloch sphere) is induced by a resonant field driving transitions between these eigenstates. In classical terms, it corresponds to a rotation of the arrow along a meridian from the north to the south pole and back. In quantum terms, at each stage of the rotation, the system is in a spin coherent state ${ }^{16}$, superposition of $|J, J-k\rangle$ s, the average value of $J-k$ coinciding with the projection of the arrow on the polar axis.

Repeatedly measuring the value of this projection would freeze the rotation, merely realizing the quantum Zeno effect. Here, instead, we implement the QZD by applying continuously a selective unitary evolution addressing only one of the $|J, J-k\rangle$ states. This state corresponds to a well-defined 'limiting latitude' on the Bloch sphere. The spin is forbidden to cross the limiting latitude and its motion remains confined on the north polar cap ${ }^{5}$.

This confined motion is non-trivial. As the rotating spin reaches the limiting latitude crossing point, it vanishes suddenly and reappears at a point on the limiting latitude with opposite longitude (inversion of the spin's azimuthal phase). The rotation then resumes towards the north pole. The complete, smooth rotation of the classical dynamics is interrupted by sudden phase inversions and replaced by a confined motion on the polar cap bounded by the limiting latitude. Caught at the phase inversion time, the spin is transiently in a quantum superposition of two spin coherent states pointing along opposite longitudes-a cat state.

This confined evolution is similar to that predicted for QZD in the cavity or circuit quantum electrodynamics context ${ }^{4,5}$. The dynamics of an angular momentum near the north pole of the Bloch sphere is analogous to that of a one-dimensional field oscillator, with $k$ playing the role of the photon number ${ }^{12}$. In this analogy, the polar cap of the Bloch sphere becomes the phase plane spanned by the field quadratures. Our experiment can thus be viewed as a quantum simulation of the cavity quantum electrodynamics version of QZD.

The spin $J=25$ is implemented in a subspace of the Stark manifold of a Rydberg atom. The interest of coherent manipulations of Rydberg manifolds has already been demonstrated in pioneering experiments on coherent wave packet dynamics ${ }^{17-19}$. We take advantage of the versatility of this system to demonstrate here a new quantum feature. Figure 1a sketches parts of three adjacent Rydberg manifolds ${ }^{20}$ (principal quantum numbers $n_{f}=52, n_{e}=51$ and $n_{g}=50$ ) in a static electric field $\mathbf{F}$, defining the quantization axis $\mathrm{Oz}$. The eigenstates are sorted in columns according to their magnetic quantum number $m$ (selected to be positive). The circular state ${ }^{21,22}$ in the $n_{e}$ manifold (thickest line) has the maximum allowed $m=n_{e}-1$ value. A $\sigma_{+}$-polarized radiofrequency (RF) field couples it to a ladder of nearly equidistant levels (thick lines). The transitions 


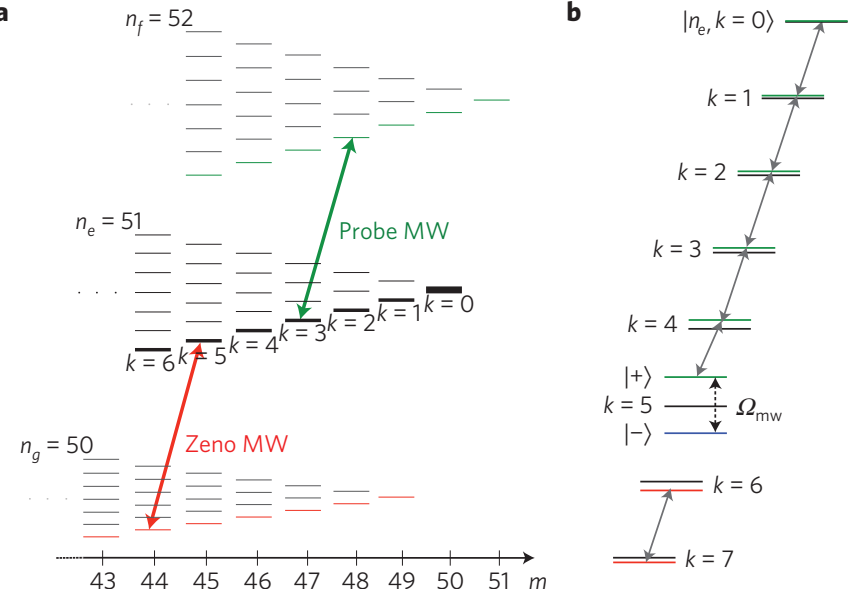

Figure 1 | Rydberg energy levels. a, Stark manifolds with principal quantum numbers $n_{g}=50, n_{e}=51$ and $n_{f}=52$ (not to scale). In each manifold, the levels, sorted by their magnetic quantum number $m$, form a triangular pattern. The thick lines represent the spin states ladder coupled to the circular state $\left|n_{e}, 0\right\rangle$ (thickest line) by the $\sigma_{+}$RF field. The green and red arrows show the transitions resonant with the probe pulse used for the spin state population measurement (here for $k_{p}=3$, see Methods) and with the Zeno microwave field (MW) for $k_{z}=5$ respectively. $\mathbf{b}$, Spin states without (black lines) and with (coloured lines) the Zeno MW. The Zeno field splits the $\left|n_{e}, k_{z}\right\rangle$ state into two dressed states, $|+\rangle$ (green line) and $|-\rangle$ (blue line), separating the Hilbert space into two subspaces $\mathcal{H}_{\mathcal{N}}$ and $\mathcal{H}_{\mathcal{S}}$ (spanned by green and red states respectively). The RF field induces transitions within these subspaces (tilted arrows) but is unable to connect them through the gap $\Omega_{\mathrm{mw}}$ opened by the Zeno MW. The Rabi splitting and the light shifts are exaggerated for clarity.

between adjacent ladder states are at the Stark angular frequency $\omega_{a}=(3 / 2) n_{e} e a_{0} F / \hbar$ within small second-order corrections in $F\left(a_{0}\right.$ : Bohr radius, $e$ : charge quantum). Because the atom is prepared initially in the circular state, the other levels in the manifold are not populated by the RF-induced dynamics and are ignored. The atom evolves within a ladder of 51 levels, $\left|n_{e}, k=0\right\rangle \ldots\left|n_{e}, k=50\right\rangle$, where $\left|n_{e}, k=0\right\rangle$ is the circular state.

The coherent evolution induced by the RF field is ruled by the Hamiltonian ${ }^{23}$ :

$$
\hat{V}=\frac{\hbar \Omega_{\mathrm{rf}}}{2} \sum_{k} \sqrt{(k+1)\left(n_{e}-k-1\right)}\left|n_{e}, k+1\right\rangle\left\langle n_{e}, k\right|+\text { h.c. }
$$

This Hamiltonian describes the rotation of a $J=25$ angular momentum at a Rabi frequency $\Omega_{\mathrm{rf}}$ (ref. 16), with the correspondence $\left|n_{e}, k\right\rangle \rightarrow|J, J-k\rangle$.

The atomic state, driven by the RF, moves down and up the ladder, whereas the equivalent angular momentum rotates around a meridian of its Bloch sphere. We observe this rotation by applying the resonant RF for a time $t_{1}$ and then measuring the populations of $\left|n_{e}, k\right\rangle$ as a function of $t_{1}$ by field ionization (Methods). Figure 2a shows $P\left(k, t_{1}\right)(k=0 \ldots 5)$ versus $t_{1}$, for $F=2.35 \mathrm{~V} \mathrm{~cm}^{-1}$, corresponding to $\omega_{a} / 2 \pi=230.15 \mathrm{MHz}$ and $\Omega_{\mathrm{rf}} / 2 \pi=152 \pm 4 \mathrm{kHz}$. The conspicuous cascade down the state ladder reveals the spin rotation. The insets show snapshots of the population distribution in the ladder levels. Data are in excellent agreement with the theoretical expectations for a rotating spin coherent state ${ }^{24}$.

To induce the QZD, we continuously interrogate the atom by selectively addressing one of the spin states with a 'Zeno' continuous wave microwave field (MW) resonant on the transition $\left|n_{e}, k_{z}\right\rangle \rightarrow\left|n_{g}, k_{z}\right\rangle$ (red arrow on Fig. 1a). For levels $\left|n_{e}, k \neq k_{z}\right\rangle$, this $\mathrm{MW}$ is non-resonant and produces only small light shifts. For $k=k_{z}$,

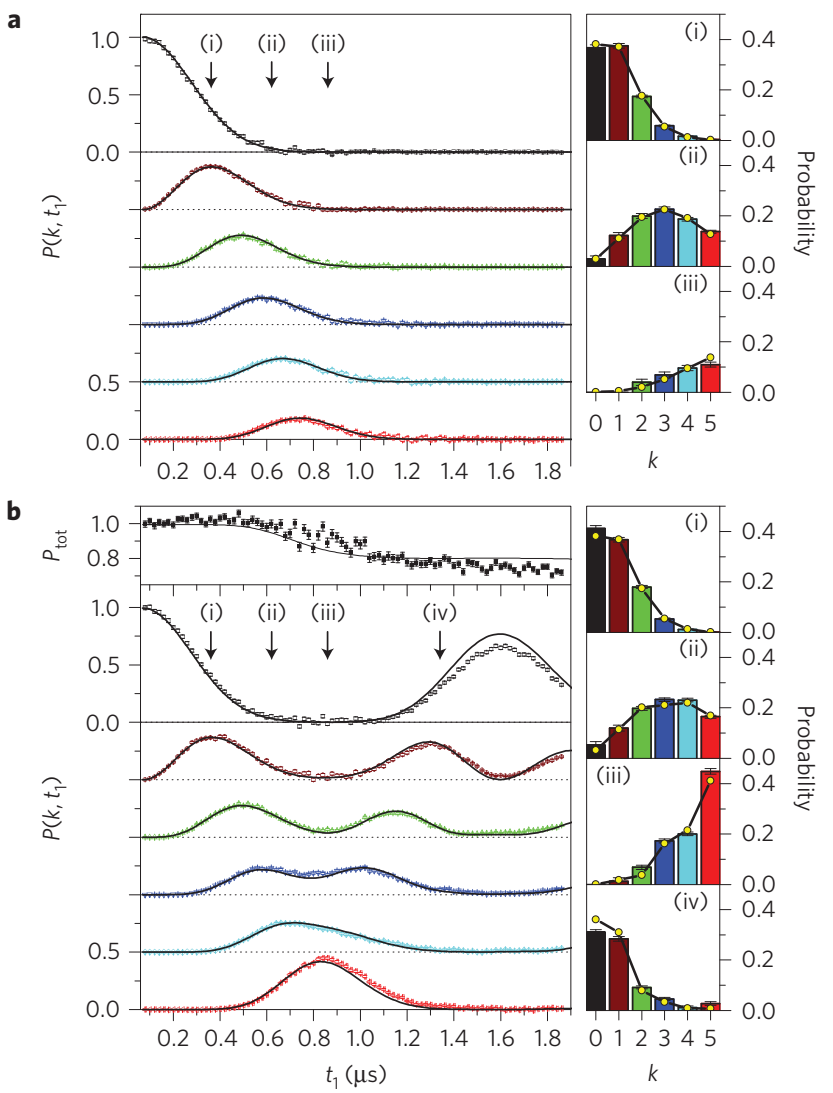

Figure 2 | Evolution of the spin state populations. a, $P\left(k, t_{1}\right)$ for $k \leq 5$ in a free RF-induced rotation (no Zeno MW applied). The points are experimental with error bars (standard deviation) reflecting the statistical nature of the detection process. The solid lines correspond to the theoretical expectation for a spin coherent state rotating at the fitted frequency $\Omega_{\mathrm{rf}}$. The insets define the colour code for the different $k$ values and present the experimental histograms of $P\left(k, t_{1}\right)$ for three $t_{1}$ values (arrows in the main frame), together with the expected spin coherent state distribution (solid lines with yellow dots). b, $P\left(k, t_{1}\right)$ for $k \leq 5$ in a QZD with $k_{z}=5$. The top frame gives the total population $P_{\text {tot }}$ of $\mathcal{H}_{\mathcal{N}}$. The lines result from the complete numerical simulation of the experiment (Methods). The insets give the observed $P\left(k, t_{1}\right)$ distribution at four $t_{1}$ values (arrows in the main frame) together with the numerical predictions (solid lines with yellow dots). Each point corresponds to $\sim 3,000$ repetitions of the experiment.

the Zeno MW admixes $\left|n_{g}, k_{z}\right\rangle$ with $\left|n_{e}, k_{z}\right\rangle$ replaced by a pair of dressed states, $| \pm\rangle$, separated by $\Omega_{\mathrm{mw}}$ (dynamical Stark splitting).

The resulting level ladder is sketched in Fig. 1b. The $\sigma_{+}$ transitions within the subspace $\left\{\left|n_{e}, 0\right\rangle,\left|n_{e}, 1\right\rangle, \ldots\left|n_{e}, k_{z}-1\right\rangle,|+\rangle\right\}$ (arrows in Fig. 1b) are nearly degenerate at the frequency $\omega_{a}$. The Zeno MW dressing opens, between $|+\rangle$ and $|-\rangle$, a gap wider than the coupling matrix element $\sim \sqrt{n_{e}} \Omega_{\mathrm{rf}}$ of $\hat{V}$ between the spin states. It makes it nearly impossible for the RF drive to induce, in an evolution from $\left|n_{e}, 0\right\rangle$, transitions towards states below $|+\rangle$. The population of $|-\rangle$ is negligible and this state can be disregarded in the discussion. Moreover, after an appropriate adiabatic switchingoff of the Zeno MW (Supplementary Information), $|+\rangle$ is mapped onto $\left|n_{e}, k_{z}\right\rangle\left(|-\rangle\right.$, being mapped onto $\left.\left|n_{g}, k_{z}\right\rangle\right)$. The QZD thus splits the angular momentum Hilbert space into $\mathcal{H}_{N}$, made up of the $k_{z}+1$ levels with $k \leq k_{z}$ close to the north pole of the Bloch sphere, and the complementary southern subspace $\mathcal{H}_{S}\left(k>k_{z}\right)$.

After a RF-induced QZD lasting a time $t_{1}$, we probe the level populations in $\mathcal{H}_{N}$. We adiabatically switch off the Zeno MW and measure $P\left(k, t_{1}\right)$. Figure $2 \mathrm{~b}$ presents the results of this procedure for $k_{z}=5$ and $\Omega_{\mathrm{mw}} / 2 \pi=3.4 \mathrm{MHz}$. The state distribution now bounces 


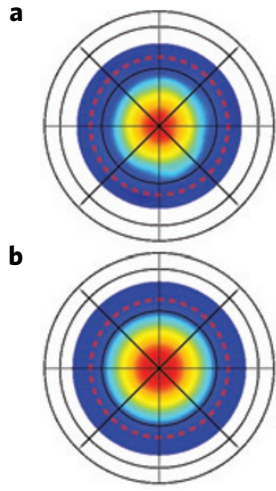

No RF
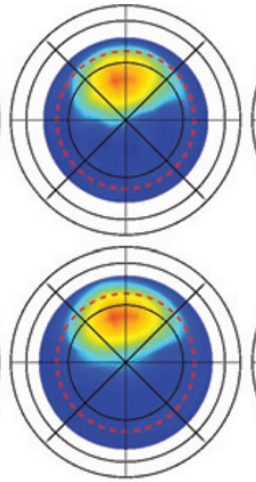

$t_{1}=0.50 \mu \mathrm{s}$

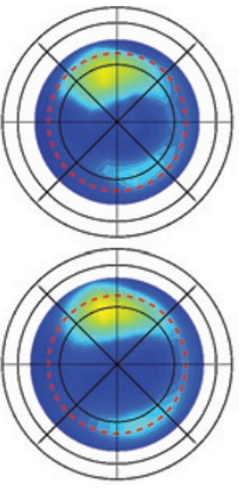

$t_{1}=0.68 \mu \mathrm{s}$

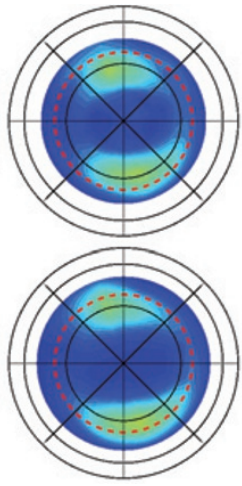

$t_{1}=0.76 \mu \mathrm{s}$

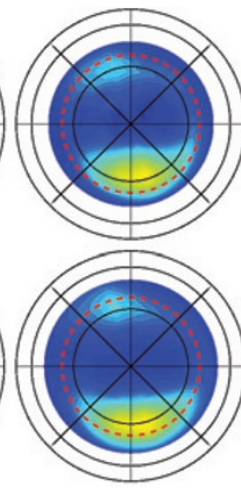

$t_{1}=0.83 \mu \mathrm{s}$
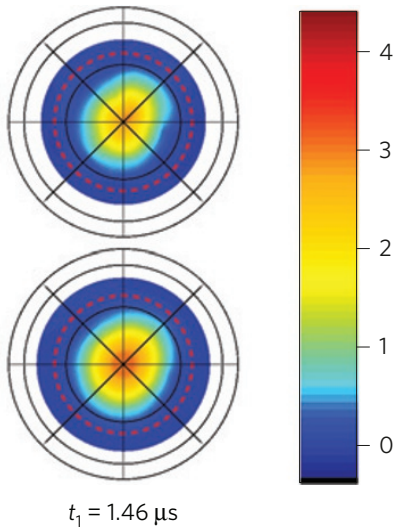

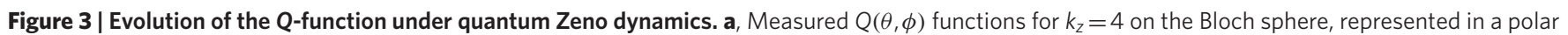
projection. The black thin lines indicate three parallels, separated by 30 degrees, and eight meridians. The data are linearly interpolated from 96 measurements points at different $(\theta, \phi)$ values spanning the north polar cap. Left frame: initial $\left|n_{e}, k=0\right\rangle$ state (no RF rotation). The other frames correspond to increasing times $t_{1}$ from left to right. The motion is confined by Zeno dynamics inside the limiting latitude (dashed red circle). The slight anticlockwise rotation of the state is due to the differential light shifts induced by the Zeno MW. b, Corresponding numerical predictions.

off a 'wall' at $k=k_{z}+1$ and nearly returns into the initial state after $1.6 \mu$ s. This dynamics is drastically different from the runaway process observed without Zeno MW (Fig. 2a). It is in excellent agreement with a complete numerical simulation of the experiment based on the independently measured experimental parameters (Supplementary Information).

The top frame in Fig. 2b shows the total population detected in $\mathcal{H}_{\mathcal{N}}$. It drops by $\sim 25 \%$ at the bouncing time. This loss is mainly due to a residual transfer into $\mathcal{H}_{\mathcal{S}}$ through the Zeno barrier. The insets show the histograms of $P\left(k, t_{1}\right)$ at four different times. As $t_{1}$ increases, the $P\left(k, t_{1}\right)$ values become radically different from those obtained without QZD (Fig. 1a). We can clearly see that the level population at the bouncing time is no longer that of a coherent spin state. Not only does QZD restrict the evolution to a subspace of five states instead of 51, but the dynamics itself exhibits striking nonclassical features.

We get a clearer picture of this dynamics by a direct measurement of the spin's $Q$-function ${ }^{25}$, transposing to spin systems the quantum optics Husimi distribution. It is defined on the Bloch sphere as $Q(\theta, \phi)=(2 J+1) /(4 \pi)\left\langle n_{e}, 0\left|R^{\dagger}(\theta, \phi) \rho R(\theta, \phi)\right| n_{e}, 0\right\rangle$, where $\rho$ is the angular momentum density operator and $R$ the rotation along a meridian of the Bloch sphere bringing the north pole to the direction defined by the polar angles $\theta$ and $\phi$.

Determining $Q$ thus amounts to measuring the population in $\left|n_{e}, 0\right\rangle$ after rotating the state by means of a resonant RF pulse whose duration $t_{2}$ controls $\theta$ and whose adjustable phase controls $\phi$. We perform this rotation with a RF power much larger than that used for the QZD (coupling $\sqrt{n} \Omega_{\mathrm{rf}}^{\prime}=2 \pi \times 6.3 \mathrm{MHz}>\Omega_{\mathrm{mw}}$ ). It couples $\mathcal{H}_{N}$ and $\mathcal{H}_{\mathcal{S}}$ even in the presence of the Zeno MW (Supplementary Information).

Figure 3a shows six snapshots of $Q$ for $k_{z}=4$ and $\Omega_{\mathrm{mw}} / 2 \pi=3.08 \pm 0.11 \mathrm{MHz}$. The initial $\left|n_{e}, 0\right\rangle$ state has a Gaussian Q-function centred at the north pole, which first moves, upwards in Fig. 3, towards the limiting latitude (dashed red line). It then splits into two components with opposite azimuthal phases. The upper component rapidly decreases, whereas the lower component grows. At $t_{1}=0.76 \mu$ s the two peaks are balanced (fourth frame). After the phase inversion, the Q-function is mainly located in the lower part of the limiting latitude and resumes its motion towards the north pole, reached again at $t_{1}=1.46 \mu$ s (last frame). Figure $3 \mathrm{~b}$ presents the results of the full numerical simulation. The excellent agreement between simulation and experiment confirms our understanding of the system Zeno dynamics and of the spin state measurement process. The observed evolution, especially the phase inversion, is very similar to the prediction of ref. 4 in the cavity quantum electrodynamics context. The fact that here the atom can populate the barrier state $|+\rangle$ does not qualitatively change the dynamics.

At $t_{1}=0.76 \mu \mathrm{s}$, we expect the system to be in a superposition of two spin coherent states with opposite azimuthal phases. However, the coherence of this superposition is not conspicuous in the $Q$-function. To get this information, we reconstruct the full angular momentum density matrix, $\rho$, at this time, through a maximum likelihood $\operatorname{method}^{26}$. It is based on the measurement of the population of several levels after adjustable RF-induced rotations and adiabatic switching-off of the Zeno MW (Methods).

Figure $4 \mathrm{a}$ shows, on the Bloch sphere, the corresponding angular momentum Wigner function ${ }^{27} W(\theta, \phi)$ at $t_{1}=0.76 \mu \mathrm{s}$. As in the quantum optics context, negative values for this quasiprobability distribution are an unambiguous indication of the state non-classicality. We observe two positive maxima near the limiting latitude. They correspond to the two spin coherent statelike components pointing towards opposite azimuthal phases at the phase inversion time. In between, the interference fringes with their negativite values give vivid evidence that we prepare a genuine quantum superposition of two distinct mesoscopic spin states-a cat state. These interference patterns cannot be observed when the Zeno subspace is only of dimension two (ref. 14). Figure $4 \mathrm{~b}$ presents the simulated Wigner function, taking into account the exact Hamiltonian of the system and all the known imperfections. Experiment and simulation are in excellent agreement (mutual fidelity 0.93 ). The measured state has a purity $\operatorname{Tr} \rho^{2}=0.75$ (simulation: 0.91). It is limited by inhomogeneities of the static electric field.

This experiment demonstrates the implementation of QZD in a Hilbert space large enough to allow us to generate mesoscopic superposition states. This is a significant step towards quantum control through Hilbert space engineering. It has been shown that the quantum control of the massively multi-level Rydberg states structure leads to important applications in state tailoring ${ }^{17,19}$ and quantum information ${ }^{18}$. The QZD opens an easily tailorable route towards the generation of such states. Moreover, the concepts and techniques used here are of general interest $t^{6-11}$ and could be applied, for instance, to superconducting qubits in circuit quantum electrodynamics (ref. 13), with direct applications to quantum information processing ${ }^{28}$.

Improving the homogeneity of the electric field would allow us to observe QZD on a longer timescale. We could perform 


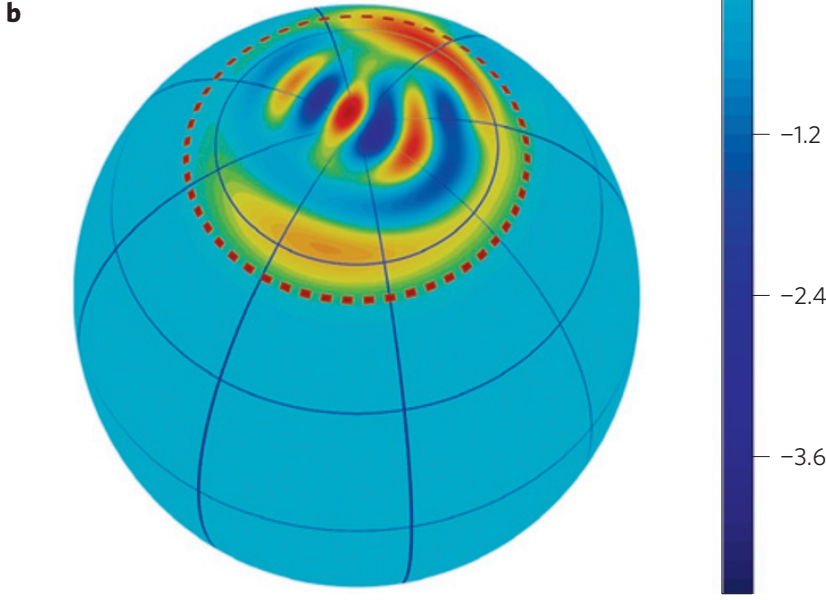

Figure $\mathbf{4}$ | Wigner function of the spin cat state. a, Experimental Wigner function, $W(\theta, \phi)$, obtained from the reconstructed density matrix $\rho$ of the spin after QZD at the phase inversion time $t_{1}=0.76 \mu$ s (corresponding to the fourth frame in Fig. 3). The interference pattern between the two classical components reveals the non-classical coherence of this state b, Result of the numerical simulation of the experiment. The fidelity of the calculated density matrix $\rho_{c}$ with $\rho$ is $\operatorname{Tr}^{2}\left(\sqrt{\sqrt{\rho} \rho_{c} \sqrt{\rho}}\right)=0.93$.

the experiment for a smaller value $\Omega_{\mathrm{rf}}$, which would reduce the leakage through the Zeno barrier, improving the fidelity of the cat state generation, and extend these experiments to larger $k_{z}$ values to prepare larger Schrödinger cat states. This opens the way to metrology beyond the standard quantum limit. The fast oscillations of the Wigner function near the north pole makes the measurement of its value a signal which is very sensitive to small rotations ${ }^{13}$. Such states could therefore be used as a very sensitive probe of small static magnetic or electric fields.

We also plan to investigate engineered decoherence ${ }^{29}$, through the application of a controlled electric field noise. The rich level structure of the Rydberg manifolds opens the way to the implementation of decoherence-free qubits through level-dressing schemes $^{30}$. Furthermore, the atomic state could be mapped onto that of a high- $Q$ cavity by tuning selected transitions in resonance by means of the Stark effect. The realization of a few-qubits processor with a single multi-level atom in a cavity is within reach.

\section{Methods}

The atoms are produced by excitation of a thermal rubidium beam. Two electrodes $A$ and $B$ facing each other (diameter $60 \mathrm{~mm}$ ) produce the directing electric field $\mathbf{F}$ along $O z$. The gap between $A$ and $B$ is surrounded by four independent electrodes, on which we apply RF signals to produce $\sigma_{+}$fields with tunable phase and amplitude.
The experimental sequence duration is $35 \mu$ s. First, the atomic sample (1.5 atoms on the average) is prepared in the circular Rydberg state by pulsed laser excitation followed by an RF-induced adiabatic rapid passage through the spin ladder. The whole process is completed in $5.6 \mu$ s. Doppler-selective laser excitation addresses atoms with a velocity $v=254 \pm 4 \mathrm{~m} \mathrm{~s}^{-1}$.

The residual static field inhomogeneities and the atomic motion limit the useful observation time. Therefore the QZD itself lasts at most $3 \mu$ s, with a first RF pulse of duration $t_{1}$, eventually followed by a second RF rotation for the state reconstruction experiments. The Zeno MW is then adiabatically switched off, and the atoms fly towards the field-ionization detector $D$ outside the electrode structure.

Our detector resolves states in adjacent Rydberg manifolds, but does not resolve directly the $\left|n_{e}, k\right\rangle$ states. Hence the population $P\left(k_{p}, t_{1}\right)$ of $\left|n_{e}, k_{p}\right\rangle$ is measured by applying, before field ionization, a resonant $\pi$-microwave (MW) pulse tuned to the $\left|n_{e}, k_{p}\right\rangle \rightarrow\left|n_{f}, k_{p}\right\rangle$ transition (green arrow on Fig. 1a). This pulse does not address the levels $\left|n_{e}, k \neq k_{p}\right\rangle$, owing to the difference between the linear Stark frequencies in adjacent manifolds. Field ionization selectively measures the population of $\left|n_{f}, k_{p}\right\rangle$, equal to $P\left(k_{p}, t_{1}\right)$ within the $\pi$-pulse transfer efficiency, $\eta_{k_{p}} \sim 0.9$ (Supplementary Information).

All parameters of the experiment are independently measured or extracted from fits between the data and a numerical simulation of the experiment, taking into account the complete level structure.

The Wigner function measurement is based on a complete reconstruction of the atomic state $\rho_{t}$ in the $n_{g}$ and $n_{e}$ manifolds. Measurements of the populations of the $\left|n_{g}, k\right\rangle$ and $\left|n_{e}, k\right\rangle(k<6)$ levels after a rotation of the spin states are used to fit $\rho_{t}$ using a maximum likelihood procedure. The final result is then projected onto the spin state ladder. This approach leads to a direct calibration of the experimental imperfections: approximately $9.3 \%$ of the population is spuriously transferred in levels outside the union of $\mathcal{H}_{N}$ and $\mathcal{H}_{S}$.

Received 7 April 2014; accepted 23 July 2014; published online 31 August 2014

\section{References}

1. Misra, B. \& Sudarshan, E. C. G. The Zeno's paradox in quantum theory. J. Math. Phys. 18, 756-763 (1977).

2. Facchi, P. \& Pascazio, S. Quantum Zeno subspaces. Phys. Rev. Lett. 89, 080401 (2002).

3. Facchi, P. \& Pascazio, S. Quantum Zeno dynamics: Mathematical and physical aspects. J. Phys. A 41, 493001 (2008).

4. Raimond, J. M. et al. Phase space tweezers for tailoring cavity fields by quantum Zeno dynamics. Phys. Rev. Lett. 105, 213601 (2010).

5. Raimond, J. M. et al. Quantum Zeno dynamics of a field in a cavity. Phys. Rev. A 86, 032120 (2012).

6. Wang, X. B., You, J. Q. \& Nori, F. Quantum entanglement via two-qubit quantum Zeno dynamics. Phys. Rev. A 77, 062339 (2008).

7. Maniscalco, S., Francica, F., Zaffino, R. L., Lo Gullo, N. \& Plastina, F. Protecting entanglement via the quantum Zeno effect. Phys. Rev. Lett. 100, 090503 (2008).

8. Rossi, R., Fonseca Romero, K. M. \& Nemes, M. C. Semiclassical dynamics from Zeno-like measurements. Phys. Lett. A 374, 158-160 (2009).

9. Shao, X. Q., Chen, L., Zhang, S. \& Yeon, K-H. Fast CNOT gate via quantum Zeno dynamics. J. Phys. B 42, 165507 (2009).

10. Chandrashekar, C. M. Zeno subspace in quantum-walk dynamics. Phys. Rev. A 82, 052108 (2010).

11. Shi, Z. C., Xia, Y., Wu, H. Z. \& Song, J. One-step preparation of three-particle Greenberger-Horne-Zeilinger state via quantum Zeno dynamics. Eur. Phys. J. D 66, 127 (2012).

12. Haroche, S. \& Raimond, J. M. Exploring the Quantum (Oxford Univ. Press, 2006).

13. Vlastakis, B. et al. Deterministically encoding quantum information using 100-photon Schrödinger cat states. Science 342, 607-610 (2013).

14. Schäfer, F. et al. Experimental realization of quantum zeno dynamics. Nature Commun. 5, 3194 (2014).

15. Ockeloen, C. F., Schmied, R., Riedel, M. F. \& Treutlein, P. Quantum metrology with a scanning probe atom interferometer. Phys. Rev. Lett. 111, 143001 (2013).

16. Arecchi, F., Courtens, E., Gilmore, R. \& Thomas, H. Atomic coherent spin states in quantum optics. Phys. Rev. A 6, 2211-2237 (1972).

17. Bromage, J. \& Stroud, C. R. Jr Excitation of three-dimensionally localized atomic electron wave packet. Phys. Rev. Lett. 83, 4963-4966 (1999).

18. Ahn, J., Weinacht, T. C. \& Bucksbaum, P. H. Information storage and retrieval through quantum phase. Science 287, 463-465 (2000).

19. Dunning, F. B., Mestayer, J. J., Reinhold, C. O., Yoshida, S. \& Burgdörfer, J. Engineering atomic Rydberg states with pulsed electric fields. J. Phys. B 42, 022001 (2009).

20. Gallagher, T. F. Rydberg Atoms (Cambridge Univ. Press, 1994) 
21. Hulet, R. G. \& Kleppner, D. Rydberg atoms in "circular" states. Phys. Rev. Lett. 51, 1430-1433 (1983).

22. Cheng, C. H., Lee, C. Y. \& Gallagher, T. F. Production of circular Rydberg states with circularly polarized microwave fields. Phys. Rev. Lett. 73, 3078-3081 (1994).

23. Englefield, M. J. Group Theory and the Coulomb Problem (Wiley, 1972).

24. Mogensen, K. S., Day, J. C., Ehrenreich, T., Horsdal Pedersen, E. \& Taulbjerg, K. Coherent elliptic states in lithium. Phys. Rev. A 51, 4038-4047 (1995).

25. Agarwal, G. S. State reconstruction for a collection of two-level systems. Phys. Rev. A 57, 671-673 (1998)

26. Lvovsky, A. I. Iterative maximum-likelihood reconstruction in quantum homodyne tomography. J. Opt. B 6, S556-S559 (2004).

27. Dowling, J. P., Agarwal, G. S. \& Schleich, W. P. Wigner distribution of a general angular-momentum state: Applications to a collection of two-level atoms. Phys. Rev. A 49, 4101-4109 (1994)

28. Massar, S. \& Polzik, E. S. Generating a superposition of spin states in an atomic ensemble. Phys. Rev. Lett. 91, 060401 (2003).

29. Poyatos, J. F., Cirac, J. I. \& Zoller, P. Quantum reservoir engineering with laser cooled trapped ions. Phys. Rev. Lett. 77, 4728-4731 (1996)

30. Aharon, N., Drewsen, M. \& Retzker, A. General scheme for the construction of a protected qubit subspace. Phys. Rev. Lett. 111, 230507 (2013).

\section{Acknowledgements}

We thank S. Pascazio and P. Facchi for many discussions and fruitful exchanges. We acknowledge funding by ANR under the project 'QUSCO-INCA' and by the EU under the ERC project 'DECLIC'

\section{Author contributions}

All authors contributed to the experimental set-up. A.S., A.F. and D.G. collected the data and analysed the results. J-M.R., S.H. and M.B. supervised the research. S.G. led the experiment and performed the numerical simulations. All authors discussed the results and the manuscript.

\section{Additional information}

Supplementary information is available in the online version of the paper. Reprints and permissions information is available online at www.nature.com/reprints.

Correspondence and requests for materials should be addressed to S.H.

\section{Competing financial interests}

The authors declare no competing financial interests. 